\title{
Static Analysis of Buried Pipes Using Coupling between Layerwise Finite Element and Boundary Element Method
}

\author{
Raimundo A. Menezes Junior and Angelo Vieira Mendonça \\ Department of Mechanical Engineering, Federal University of Paraiba, João Pessoa 58059-900, Brazil
}

Received: March 01, 2014 / Accepted: March 21, 2014 / Published: September 25, 2014.

\begin{abstract}
This work deals with the analysis of soil-structure interaction modeling of pipeline problems in static behavior using the coupling between FEM (finite element method) and BEM (boundary element method). The representation of the pipe is made by MEF using one finite element in the cylindrical panel formulated from the theory of equivalent discrete layers (Layerwise theory), proposed by J. N. Reddy. The soil is represented by elastic continum infinite or semi-infinite and modeled using boundary elements with special curved surface, associated with cylindrical panel used to represent the soil-structure interaction within the soil, especially at the contact surface with the pipe.
\end{abstract}

Key words: Finite elements, boundary elements, pipe-soil coupling.

\section{Introduction}

In recent years, many sectors in the industry have implements and improve their infrastructures with the use of buried and submerged pipelines. These structures to be interacted with other media (soil, liquid, etc.) have a high degree of complexity, which demands special attention from initial conception until the final operation. Thus, in recent decades, designers have used some tools to help to produce projects of buried pipelines more efficient. One of the important lines is based on the accumulated experience that is usually described in norms already established. However, this is not enough, since each project has its own peculiarities. Then, in many cases, a set of experimental tests are specified to extract some behavior parameters of the buried pipe. However, even with the great help of the experimental apparatus, there are important unknowns in problem behavior that are generally not accessible appropriately, such as the state of deformation and stress in the pipes and its interfaces with the ground. Thus, the third family of

Corresponding author: Raimundo A. Menezes Junior, Ph.D., research field: mechanical engineering. E-mail: jrmenezes@cear.ufpb.br. tools that can potentially be used are the numerical simulation techniques enabling the structural analysis of the problem. The mathematical models commonly used are usually restricted to two-dimensional space. If the analysis region of the line along its length is extended without significant change, the two-dimensional analysis results generally have no significant differences when compared to the three-dimensional model. However, it is not always possible to ensure the proximity of pipe remaining unchanged, as it usually has to go through urban areas, cross roads, railroads, etc. Thus, the development and implementation of three-dimensional models for the analysis of buried pipes is undoubtedly a real need for improvement of projects. In recent decades, many researchers have been dedicated to the study of mathematical models of interaction, consisting of a set of sub-models associated respectively to the pipeline, soil and their interfaces. In general, the analytical solutions of the governing equations of buried pipes are only available for some special cases of interaction. This is the case in the one-dimensional model, when it admits the buried pipes as infinite beam supported by elastic base, which solutions are found in Refs. [1, 2]. 
Already Burns and Richard [3] analyzed a pipe-soil system subjected to horizontal and vertical pressures, using the principles of continuum mechanics, where closed solutions (analytical) for a two-dimensional elastic model (plane strain condition) were developed. Hoeg and Dhar [4, 5] also presented two-dimensional analytical solutions expressed more smoothly than techniques employed in Ref. [3].

There are many semi-empirical methods which are obtained by the incorporation of some correction factors in the two-dimensional analytical solutions, which are calibrated experimentally. Among the techniques, most known semi-empirical has Iowa method [6] and its modified versions are shown in Refs. [7, 8]. However, not all the analytical solutions of pipelines are available. So an alternative is the discretization of continuous medium; and the systematization of the discrete problem is to obtain approximate solutions using numerical techniques, among which has been the FEM (finite element method) and BEM (boundary element method). In this context, for the one-dimensional model, there are some numerical solutions where the domain is usually discretized by 1D finite element (bars) and the effect of elastic foundation is introduced into the problem by potential energy. This produces an additional contribution to the stiffness matrix of the structure. For the two-dimensional model, there are several numerical solutions for pipeline and soil represented by continuous media: FEM, BEM [9] and FEM-BEM combination [10]. For numerical analysis of the three-dimensional models, some work can be found in the literature for each of the sub-systems involving isolated pipe, isolated soil and soil-pipe interaction. Numerical models for analysis already insulated idealized as shells. Several researchers [11-14, 24-26] formulated plane shells finite elements, for example, the membrane finite element called CST (constant stress triangle), combined with plate elements, such as discussed in Ref. [15] or DST (discrete shear triangular) described in Ref. [16]. A research group showed an alternative finite element family for cylindrical shells analysis called assumed strain-based model. Ref. [17] was one of the first works with analysis of deep cylindrical shells. Already Djoudi and Bahai $[18,19]$ presented an extension of the ideas of the model to cylindrical shells proposed by Sabir employing the theory of shells in Ref. [20]. Against this background, this paper presents a mathematical model for the analysis of three-dimensional problems of buried homogeneous pipes.

\section{Layerwise Cylindrical Shell Finite Element}

In this section, we describe the formulation of the laminated cylindrical shell based on Layerwise theory in Ref. [21] and systematized by some researchers [22]: Savoia and Reddy (1992) and Gerhard et al. (1994). The laminated cylindrical shell has layers with the same or different thickness, composed by materials with different orientation angles to the fibers. The representation of a cylindrical shell with length $L$, thickness $h$ and deformable surface with radius $R$ (where, $h<<R$ ) is shown in Fig. 1.

The displacement field $(u, v, w)$ at a generic point $(x$, $y, z)$ of a layer in one laminated cylindrical shell is approximated by the following Lagrangian interpolation functions [23].

$$
\begin{gathered}
u(x, y, z)=\sum_{i=1}^{n+1} u_{i}(x, y) \Phi^{i}(z) \\
v(x, y, z)=\sum_{i=1}^{n+1} v_{i}(x, y) \Phi^{i}(z) \\
w(x, y, z)=\sum_{i=1}^{n+1} w_{i}(x, y) \Phi^{i}(z)
\end{gathered}
$$

\section{Nodal Load Equivalent Vector}

The nodal load equivalent vector is deducted for uniformly and linearly distributed self-weight for the finite element RLS (Reddy laminated shell). The consistent nodal vector is obtained through the work done by external loads that can be expressed by: 


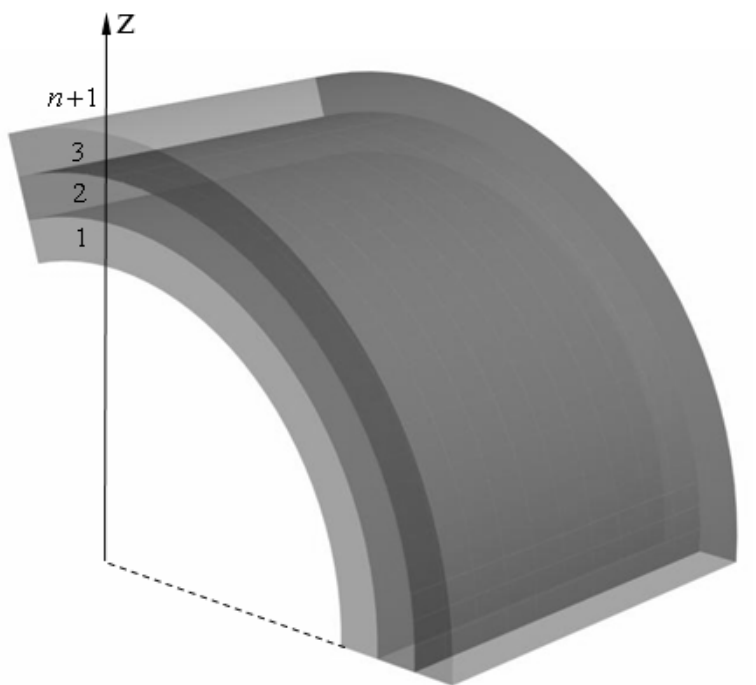

Fig. 1 Finite element of laminated cylindrical shell.

$$
W=\int_{A}\left(p_{x} u+p_{y} v+p_{z} w\right) \mathrm{d} A=\int_{A}\left\{\begin{array}{l}
u \\
v \\
w
\end{array}\right\}^{T}\left\{\begin{array}{l}
p_{x} \\
p_{y} \\
p_{z}
\end{array}\right\} \mathrm{d} A
$$

The external loads due to the linear distribution have the matrix representation as follows:

$$
\left\{\begin{array}{c}
p_{x} \\
p_{y} \\
p_{z}
\end{array}\right\}=[R]\{\hat{p}\}
$$

where, the $[R]$ matrix depends on kind of distribution of the pressure in the element with nodal forces vector.

$$
\begin{gathered}
\{\hat{p}\}^{T}=\left\{\begin{array}{llllll}
\hat{p}_{x 1} & \hat{p}_{y 1} & \hat{p}_{z 1} & \hat{p}_{x 2} & \hat{p}_{y 2} & \hat{p}_{z 2} \\
\hat{p}_{x 3} & \hat{p}_{y 3} & \hat{p}_{z 3} & \hat{p}_{x 4} & \hat{p}_{y 4} & \hat{p}_{z 4}
\end{array}\right\}
\end{gathered}
$$

Substituting the nodal force vector in Eq. (4) in the external work equation in Eq. (2), the equivalent nodal force vector $\{F\}$ is:

$$
\{F\}=\left[C_{d}\right]^{-T}\{P\}
$$

where,

$$
\begin{gathered}
\{P\}=[A]\{\hat{p}\} \\
{[A]=\int_{A}[Z]^{T}[R] d A}
\end{gathered}
$$

The $[A]$ matrix calculation in Eq. (5) results in the

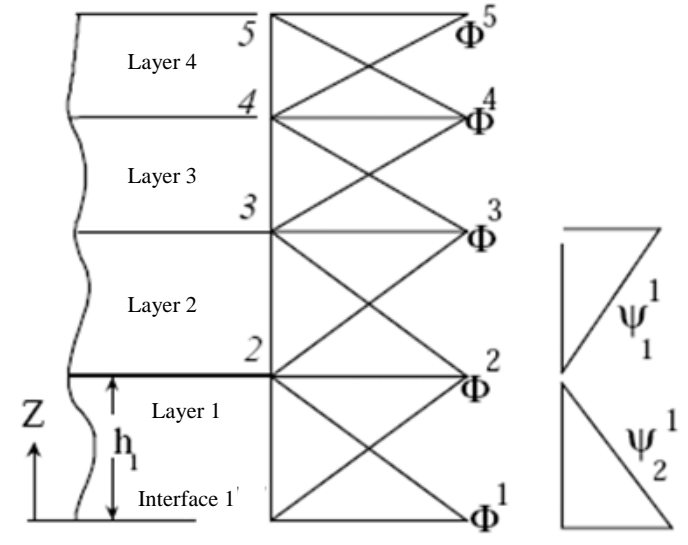

explicit form.

$$
A=\frac{a b}{9}\left[\begin{array}{cccccccccccc}
4 & 0 & 0 & 2 & 0 & 0 & 1 & 0 & 0 & 2 & 0 & 0 \\
0 & 4 & 0 & 0 & 2 & 0 & 0 & 1 & 0 & 0 & 2 & 0 \\
0 & 0 & 4 & 0 & 0 & 2 & 0 & 0 & 1 & 0 & 0 & 2 \\
2 & 0 & 0 & 4 & 0 & 0 & 2 & 0 & 0 & 1 & 0 & 0 \\
0 & 2 & 0 & 0 & 4 & 0 & 0 & 2 & 0 & 0 & 1 & 0 \\
0 & 0 & 2 & 0 & 0 & 4 & 0 & 0 & 2 & 0 & 0 & 1 \\
1 & 0 & 0 & 2 & 0 & 0 & 4 & 0 & 0 & 2 & 0 & 0 \\
0 & 1 & 0 & 0 & 2 & 0 & 0 & 4 & 0 & 0 & 2 & 0 \\
0 & 0 & 1 & 0 & 0 & 2 & 0 & 0 & 4 & 0 & 0 & 2 \\
2 & 0 & 0 & 1 & 0 & 0 & 2 & 0 & 0 & 4 & 0 & 0 \\
0 & 2 & 0 & 0 & 1 & 0 & 0 & 2 & 0 & 0 & 4 & 0 \\
0 & 0 & 2 & 0 & 0 & 1 & 0 & 0 & 2 & 0 & 0 & 4
\end{array}\right]
$$

For the case of uniform pressure in each direction, then the vector in Eq. (4) takes the following form:

$$
\begin{gathered}
\{\hat{p}\}^{T}=\left\{\begin{array}{llllll}
\hat{p}_{x 1} & \hat{p}_{y 1} & \hat{p}_{z 1} & \hat{p}_{x 2} & \hat{p}_{y 2} & \hat{p}_{z 2} \\
\hat{p}_{x 3} & \hat{p}_{y 3} & \hat{p}_{z 3} & \hat{p}_{x 4} & \hat{p}_{y 4} & \hat{p}_{z 4}
\end{array}\right\}
\end{gathered}
$$

where,

$$
\begin{gathered}
\hat{p}_{x 1}=\hat{p}_{x 2}=\hat{p}_{x 3}=\hat{p}_{x 4}=\hat{p}_{x} \\
\hat{p}_{y 1}=\hat{p}_{y 2}=\hat{p}_{y 3}=\hat{p}_{y 4}=\hat{p}_{y} \\
\hat{p}_{z 1}=\hat{p}_{z 2}=\hat{p}_{z 3}=\hat{p}_{z 4}=\hat{p}_{z}
\end{gathered}
$$

where, explicit values of subvetores $\{\hat{p}\}=\left\{\begin{array}{l}\left\{F_{1}\right\} \\ \left\{F_{2}\right\}\end{array}\right\}$ are: 


$$
\left\{F_{1}\right\}=a b\left\{\begin{array}{c}
p_{x} \\
p_{y} \\
p_{z} \\
p_{x} \\
p_{y} \\
p_{z}
\end{array}\right\} \quad\left\{F_{2}\right\}=a b\left\{\begin{array}{c}
p_{x} \\
p_{y} \\
p_{z} \\
p_{x} \\
p_{y} \\
p_{z}
\end{array}\right\}
$$

Considering the effect of the self weight of the shell divided into two stages, where, in the first, it is assumed that the longitudinal axis of the shell is orthogonal to the gravity vector, then the $[R]$ matrix in Eq. (3) takes the following form:

$$
\begin{gathered}
{[R]=\left[\begin{array}{ccc}
1 & 0 & 0 \\
0 & \cos \left(y / r+\theta_{0}\right) & 0 \\
0 & 0 & \operatorname{sen}\left(y / r+\theta_{0}\right)
\end{array}\right]} \\
\{\hat{p}\}^{T}=\left\{\begin{array}{lll}
0 & g & g
\end{array}\right\}
\end{gathered}
$$

where, $g=\gamma h, \gamma=$ specific weight.

$$
\begin{array}{r}
\text { The vector }\{\hat{p}\}=\left\{\begin{array}{l}
\left\{P_{1}\right\} \\
\left\{P_{2}\right\}
\end{array}\right\} \text { is given by: } \\
\left\{P_{1}\right\}=\left\{\begin{array}{c}
0 \\
p_{y 1} \\
p_{z 1} \\
0 \\
p_{y 1} \\
p_{z 1}
\end{array}\right\} \quad\left\{P_{2}\right\}=\left\{\begin{array}{c}
0 \\
p_{y 3} \\
p_{z 3} \\
0 \\
p_{y 3} \\
p_{z 3}
\end{array}\right\}
\end{array}
$$

where, $p_{y 1}$ to $p_{y 4}$ and $p_{z 1}$ to $p_{z 4}$ are:

$$
p_{y 1}=-\frac{r a}{2 b}\left(\begin{array}{l}
r \sin \left(\frac{b}{r}\right) \sin (\theta)- \\
b \cos \left(\frac{b}{r}\right) \sin (\theta)-b \sin \left(\frac{y}{r}\right) \cos (\theta)
\end{array}\right) \rho g t
$$$$
p_{y 1}=p_{y 2}
$$$$
p_{y 3}=-\frac{r a}{2 b}\left(\begin{array}{l}
r \sin \left(\frac{b}{r}\right) \sin (\theta)- \\
b \cos \left(\frac{b}{r}\right) \sin (\theta)+b \sin \left(\frac{y}{r}\right) \cos (\theta)
\end{array}\right) \rho g t
$$

$$
p_{y 4}=p_{y 3}
$$

$$
\begin{aligned}
& p_{z 1}=-\frac{r a}{2 b}\left(\begin{array}{l}
r \sin \left(\frac{b}{r}\right) \cos (\theta)- \\
\left.b \cos \left(\frac{b}{r}\right) \cos (\theta)-b \sin \left(\frac{y}{r}\right) \sin (\theta)\right)
\end{array}\right) \rho g t \\
& p_{z 2}=p_{z 1} \\
& p_{z 3}=-\frac{r a}{2 b}\left(\begin{array}{l}
r \sin \left(\frac{b}{r}\right) \cos (\theta)- \\
b \cos \left(\frac{b}{r}\right) \cos (\theta)-b \sin \left(\frac{y}{r}\right) \sin (\theta)
\end{array}\right) \rho g t \\
& p_{z 4}=p_{z 3}
\end{aligned}
$$

If the longitudinal axis of the shell is parallel to vector gravity, then:

$$
\left\{P_{1}\right\}=a b\left\{\begin{array}{c}
p_{x} \\
0 \\
0 \\
p_{x} \\
0 \\
0 \\
p_{x} \\
0 \\
0 \\
p_{x}
\end{array}\right\} \quad\left\{P_{2}\right\}=a b\left\{\begin{array}{c}
p_{x} \\
0 \\
0 \\
p_{x} \\
0 \\
0 \\
p_{x} \\
0 \\
0 \\
p_{x}
\end{array}\right\}
$$

where: $p_{x}=\sum \frac{\gamma_{i} \cdot t h_{i}}{2}$

\section{Usual Boundary Elements}

Triangular plans elements for discretization of contact interfaces of machine foundation and soil (Fig. 1).

The interpolation variable geometry is made linearly from three nodes functional positioned at the vertices of the triangle.

$$
\begin{aligned}
& \left\{\begin{array}{l}
u_{1} \\
u_{2} \\
u_{3}
\end{array}\right\}=\left[\begin{array}{ccc}
N_{1} & 0 & 0 \\
0 & N_{1} & 0 \\
0 & 0 & N_{1}
\end{array}\right]\left\{\begin{array}{l}
u_{1}^{1} \\
u_{2}^{1} \\
u_{3}^{1}
\end{array}\right\}+ \\
& {\left[\begin{array}{ccc}
N_{2} & 0 & 0 \\
0 & N_{2} & 0 \\
0 & 0 & N_{2}
\end{array}\right]\left\{\begin{array}{l}
u_{1}^{2} \\
u_{1}^{2} \\
u_{3}^{2}
\end{array}\right\}+\left[\begin{array}{ccc}
N_{3} & 0 & 0 \\
0 & N_{3} & 0 \\
0 & 0 & N_{3}
\end{array}\right]\left\{\begin{array}{l}
u_{1}^{3} \\
u_{1}^{3} \\
u_{3}^{3}
\end{array}\right\}}
\end{aligned}
$$




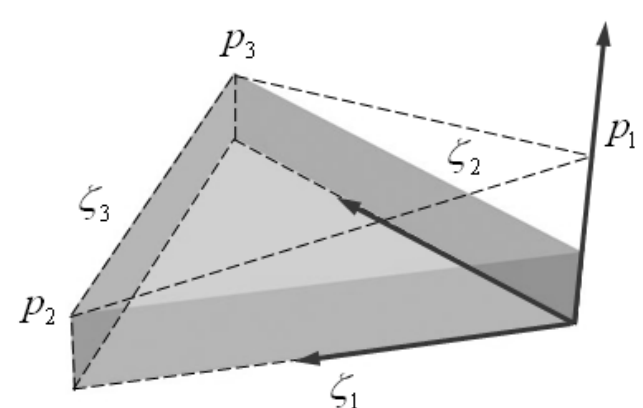

Fig. 1 Triangular boundary element.

where, the functions are:

$$
N_{1}=1-\xi-\eta ; N_{2}=\xi ; N_{3}=\eta
$$

\section{Special Boundary Elements}

In the literature (Beskos 1987), (Brebia and Dominguez 1977), most of the boundary elements are flat or approximated by polynomials, usually up to second degree. Given the specificity of the subject discussed in this work, these would not be very appropriate. Therefore, broke for the proposed new boundary element in which cylindrical surfaces, flat surfaces with polygonal boundaries could not be accurately represented. The elements proposed elements associated with a cylindrical panel to discretization the cylindrical shaft. This element had four nodal points with interpolation of variables (Fig. 2).

If considered a linear distribution of the field components within a cylindrical boundary element panel, their shape functions are interpolated:

$$
\begin{aligned}
& \left\{\begin{array}{l}
u_{x} \\
u_{\theta} \\
u_{r}
\end{array}\right\}=\left[\begin{array}{ccc}
N_{1} & 0 & 0 \\
0 & N_{1} & 0 \\
0 & 0 & N_{1}
\end{array}\right]\left\{\begin{array}{l}
u_{x}^{1} \\
u_{\theta}^{1} \\
u_{r}^{1}
\end{array}\right\}+\left[\begin{array}{ccc}
N_{2} & 0 & 0 \\
0 & N_{2} & 0 \\
0 & 0 & N_{2}
\end{array}\right]\left\{\begin{array}{l}
u_{x}^{2} \\
u_{\theta}^{2} \\
u_{r}^{2}
\end{array}\right\}+ \\
& {\left[\begin{array}{ccc}
N_{3} & 0 & 0 \\
0 & N_{3} & 0 \\
0 & 0 & N_{3}
\end{array}\right]\left\{\begin{array}{l}
u_{x}^{3} \\
u_{\theta}^{3} \\
u_{r}^{3}
\end{array}\right\}+\left[\begin{array}{ccc}
N_{4} & 0 & 0 \\
0 & N_{4} & 0 \\
0 & 0 & N_{4}
\end{array}\right]\left\{\begin{array}{l}
u_{x}^{4} \\
u_{\theta}^{4} \\
u_{r}^{4}
\end{array}\right\}}
\end{aligned}
$$

where, the interpolation functions are given by:

$$
\begin{aligned}
& N_{1}=\varepsilon_{r} \varepsilon_{\theta} ; N_{2}=\left(1-\varepsilon_{r}\right) \varepsilon_{\theta} ; \\
& N_{3}=\left(1-\varepsilon_{r}\right)\left(1-\varepsilon_{\theta}\right) ; N_{4}=\varepsilon_{r}\left(1-\varepsilon_{\theta}\right)
\end{aligned}
$$

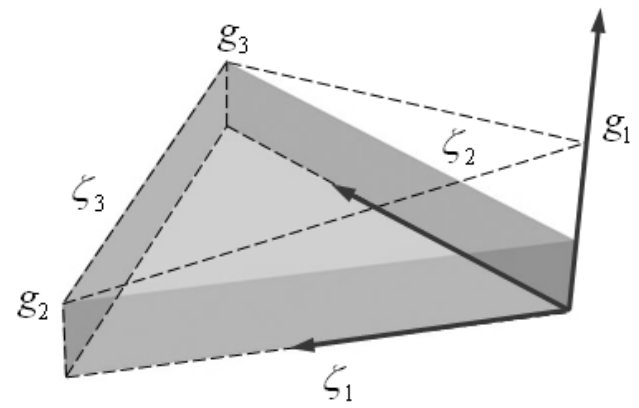

com $\varepsilon_{r}=\frac{\bar{r}}{\Delta r} ; \varepsilon_{\theta}=\frac{\bar{y}}{r \beta}=\frac{r \theta}{r \beta}=\frac{\theta}{\beta}$

Sector plans boundary elements formed by two straight sides and two curved, destined for discretization of axial extremity shaft. The fields are linearly interpolated over the nodes of the functional element (Fig. 3).

Discoid plans boundary elements, used for discretization of regions in proximity of centers of the circles in axial extremity of the shaft (Fig. 4).

Note that a single nodal point is placed at the centers.

\section{Formulation of Interaction Analysis for Soil-Pipe}

Fig. 5 presents a brief description of the problem and their interaction forces which are to be fully analyzed.

For the fundamental solution of kelvin, the $p_{i j}$ is not null in the soil region in contact with radie, which requires defining points outside the region of contact, including the boundary region for the soil that envelops a cylindrical shaft. Thus, the contact interface with the radie $(\mathrm{C})$ and the boundary contour (D) are placed sources in the shaft (E) at the end of the shaft (B). The algebraic system that represents this condition is:

$$
\left[\begin{array}{ccc}
\tilde{H}_{f f} & \tilde{H}_{f e} & \tilde{H}_{f} \\
\tilde{H}_{e f} & \tilde{H}_{e e} & \tilde{H}_{e l} \\
\tilde{H}_{l f} & \tilde{H}_{l e} & \tilde{H}_{l l}
\end{array}\right]\left\{\begin{array}{c}
\tilde{u}_{f} \\
\tilde{u}_{e} \\
\tilde{u}_{l}
\end{array}\right\}=\left[\begin{array}{ccc}
\tilde{G}_{f f} & \tilde{G}_{f e} & \tilde{G}_{f f} \\
\tilde{G}_{e f} & \tilde{G}_{e e} & \tilde{G}_{e l} \\
\tilde{G}_{l f} & \tilde{G}_{l e} & \tilde{G}_{l l}
\end{array}\right]\left\{\begin{array}{c}
\tilde{p}_{f} \\
\tilde{p}_{e} \\
\tilde{p}_{l}
\end{array}\right\}
$$




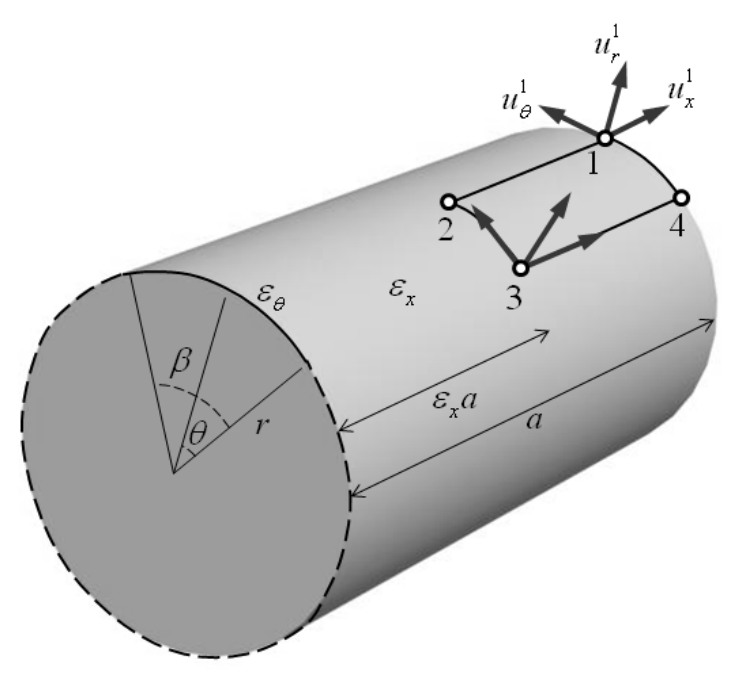

Fig. 2 Cylindrical boundary element.

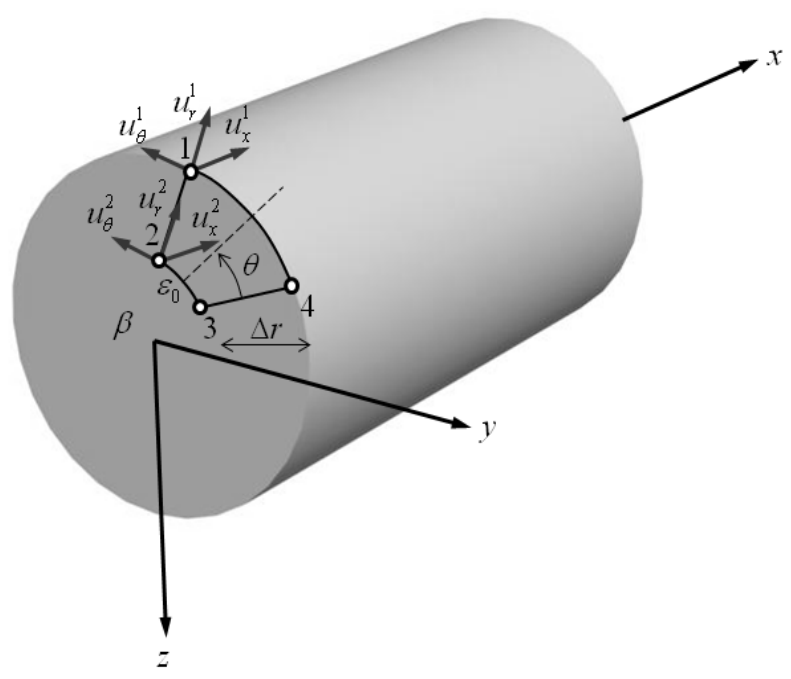

Fig. 3 Plane sector boundary element.

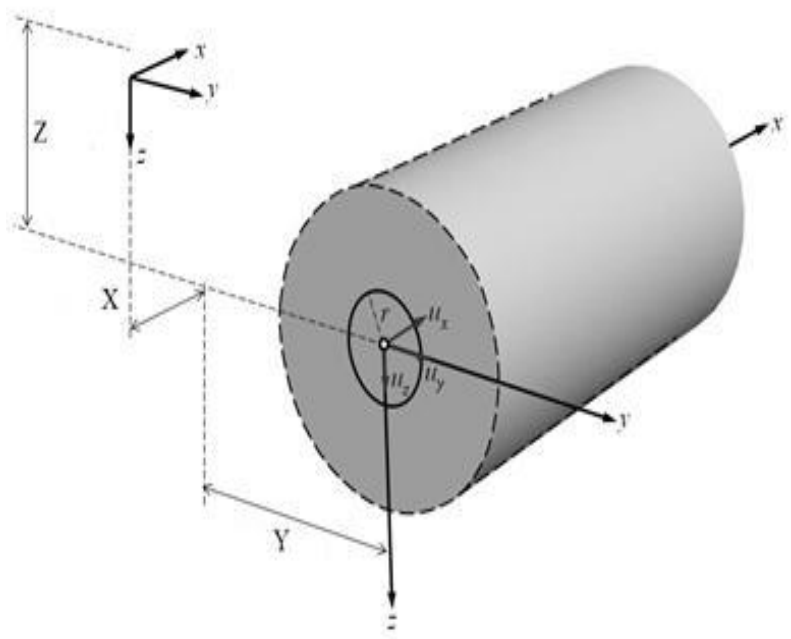

Fig. 4 Discoid boundary element.

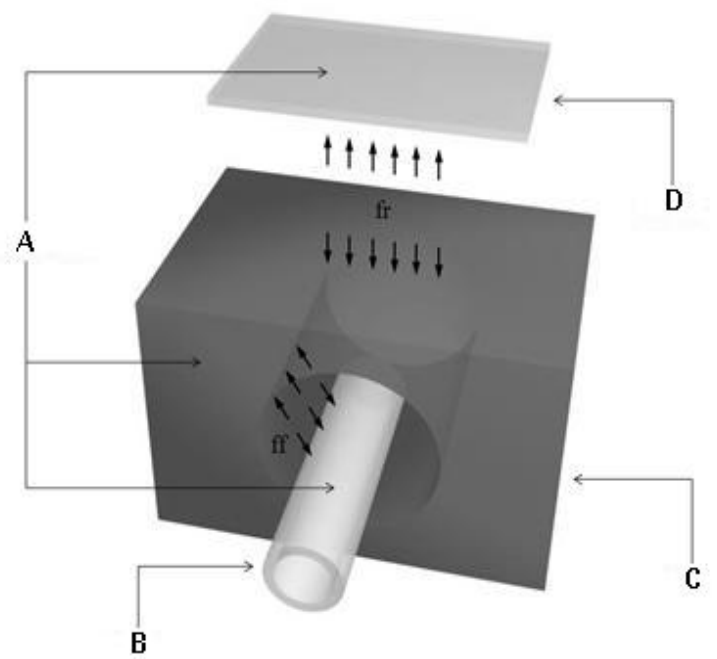

Fig. 5 Interaction forces for buried pipe problem.

A = coupling; $\mathrm{B}=$ pipe; $\mathrm{C}=$ soil and $\mathrm{D}=$ Radie

The pipe-soil interaction can be represented mathematically from the following assumptions:

- Contact between the pipe and the surface soil is ideal (there are no relative displacement at any point on the surface of interaction);

- Compatible only the degrees of freedom of displacements and forces in the axial, radial and circumferential contact surface.

The algebraic system of structure obtained from finite element discretization of cylindrical shells RLS is established with numeration of the first interface coupled with the soil, followed by the other interfaces not coupled. Thus, the system can be partitioned in the matrix form:

$$
\left[\begin{array}{ll}
\bar{K}_{L L} & \bar{K}_{L N} \\
\bar{K}_{N L} & \bar{K}_{N N}
\end{array}\right]\left\{\begin{array}{l}
\bar{U}_{L} \\
\bar{U}_{N}
\end{array}\right\}=\left\{\begin{array}{c}
\bar{F}_{L} \\
\bar{F}_{N}
\end{array}\right\}-\left\{\begin{array}{c}
P_{L} \\
0
\end{array}\right\}
$$

The configuration of degree of freedom for the finite element RLS to be coupled with boundary element of soil is shown in Fig. 6a and 7b. For the sake of conciseness, only nodes 1 and 4 are represented with the degrees of freedom associated with it.

Making the force equilibrium $\left\{\bar{P}_{L}\right\}=\left\{P_{F}\right\}$ and the compatibility of displacements $\left\{\bar{U}_{L}\right\}=\left\{U_{F}\right\}$, and substituting the soil system in Eq. (14) in the RLS 
finite element system in Eq. (15), the soil-pipe interaction is:

$$
\left[\begin{array}{cc}
{\left[\bar{K}_{L L}+K_{f f}\right]} & \bar{K}_{L N} \\
\bar{K}_{N L} & \bar{K}_{N N}
\end{array}\right]\left\{\begin{array}{l}
\bar{U}_{L} \\
\bar{U}_{N}
\end{array}\right\}=\left\{\begin{array}{l}
\bar{F}_{L} \\
\bar{F}_{N}
\end{array}\right\}+\left\{\begin{array}{c}
P_{F}^{* *} \\
0
\end{array}\right\}
$$

\section{Results}

\subsection{Analysis of a Shallow Laminated Pipe}

Whether a cavity how the radius is $2.3 \mathrm{~m}$ in a rock mass, mechanical properties are indicated in Table 1.

We considered the pipe initially homogeneous and subjected to a confining pressure due to the weight of the rock mass $P=1,000 \mathrm{kPa}$ as shown in Fig. 7 .

In table 3 , the results for the radial displacement are listed at the point of cavity 2, which are compared with analytical solution of Timoshenko (1959) and the numerical solution of BEM in Ref. [9] (using 40

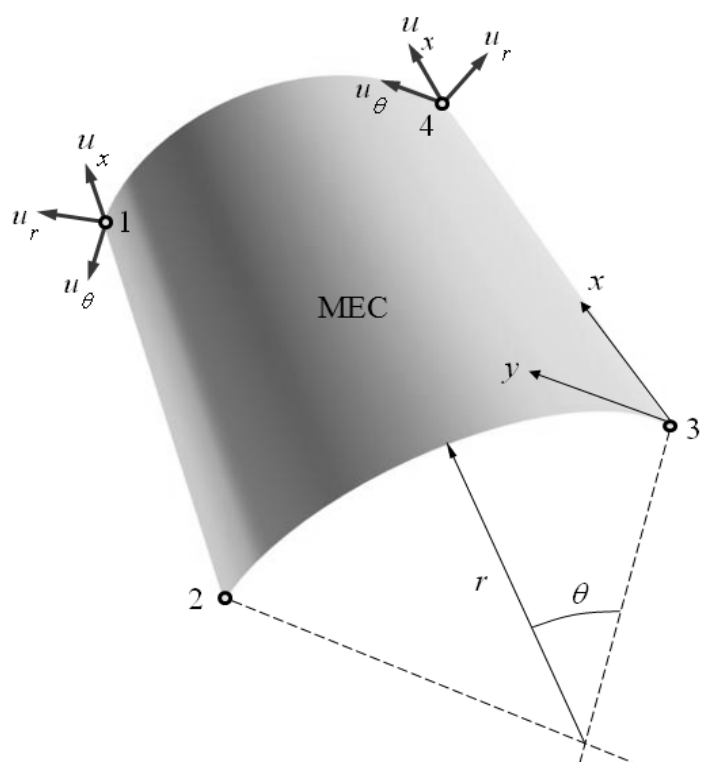

(a) elements) (table 2). In order to recover the two-dimensional analysis applying the 3D model, it was considered a shaft/cylinder with length greater than 10 times the diameter of the shaft, resulting in $L$ $=45 \mathrm{~m}$. Furthermore, displacements at the axial extremities of the shaft were prescribed. Fig. 8 describes the behavior convergence of the results for the analysis.

The results (Table 3) where RLS 2C [2 Layer] RLS 3C [3 Layer] 4C RLS [4 layers]), show that the RLS finite element, when coupled to ground, presents convergence to values the literature. It should be emphasized that the RLS element appears more effective with more layers presented in thickness of the model. However, the computational analysis becomes more costly.

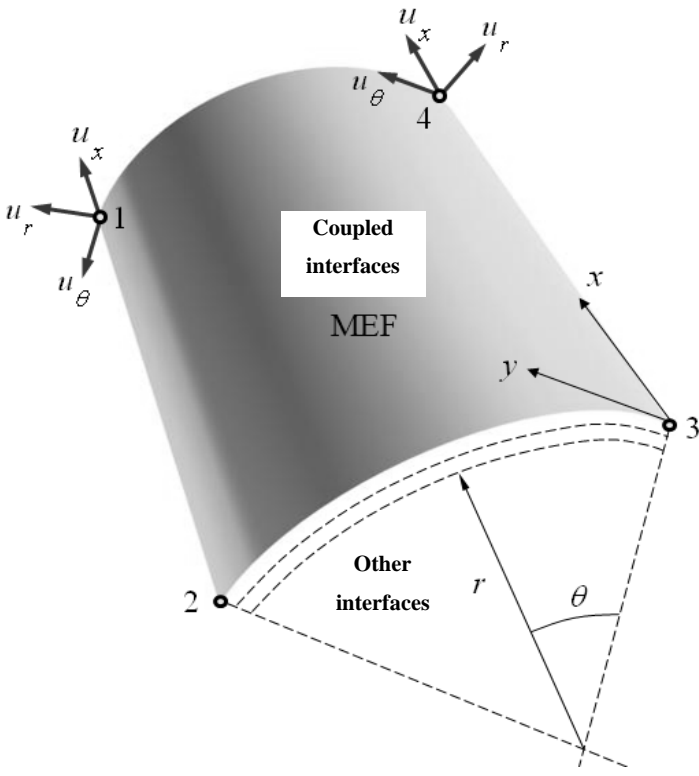

(b)

Fig. 6 (a) Soil boundary element and (b) RLS finite element.

Table 1 Physical properties of soil-pipe coupling.

\begin{tabular}{llll}
\hline E $(\mathrm{GPa})$ & $v$ & Thickness $(\mathrm{m})$ & Structure \\
\hline 12.50 & 0.20 & - & Soil \\
25.70 & 0.15 & 0.3 & Pipe \\
\hline
\end{tabular}

Table 2 Results of other authors.

\begin{tabular}{ll}
\hline Timoshenko (1959) & MEC 2D [9] \\
\hline $1.53333 \mathrm{e}-007 \mathrm{~m}$ & $1.53000 \mathrm{e}-007 \mathrm{~m}$ \\
\hline
\end{tabular}


Fig. 7 Low pressure pipe depth.
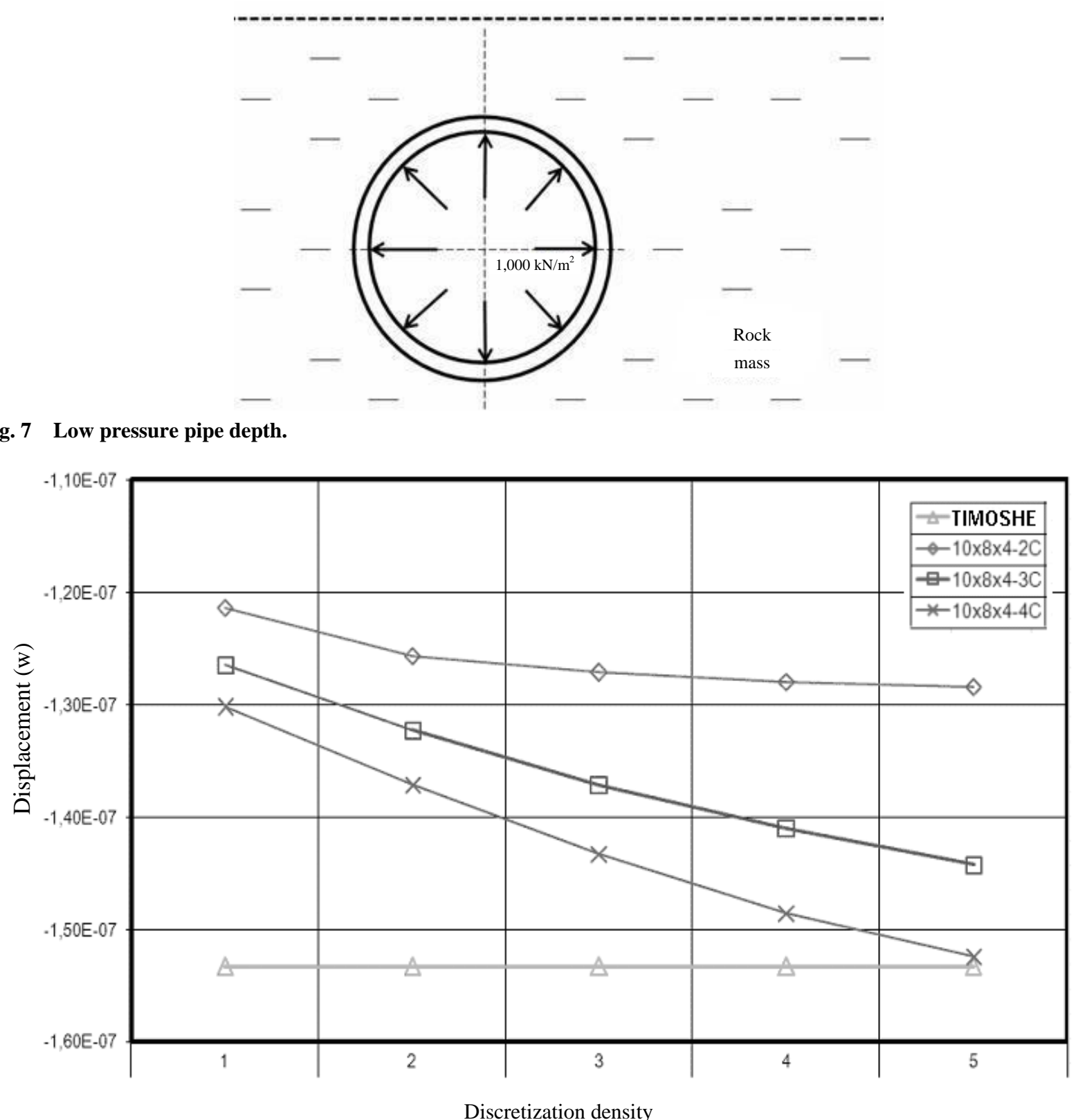

Fig. 8 Laminated low pressure pipe depth-graphic.

Table 3 Laminated low pressure pipe depth.

\begin{tabular}{lllll}
\hline Timoshe & - & $-1,533000000000000 \mathrm{e}-07$ & $-1,533000000000000 \mathrm{e}-07$ & $-1,533000000000000 \mathrm{e}-07$ \\
Mec 2d & $\square$ & $-1,517792202448180 \mathrm{e}-07$ & $-1,528000000000000 \mathrm{e}-07$ & $-1,530000000000000 \mathrm{e}-07$ \\
Mec 3d & - & $-1,480406313039020 \mathrm{e}-07$ & $-1,514863584659230 \mathrm{e}-07$ & $-1,532164517175760 \mathrm{e}-07$ \\
\hline
\end{tabular}

\section{Conclusions}

In this paper, we studied the problem analysis of insulated and buried and their neighborhood. From the standpoint of mathematical and computational, some original contributions were given and listed below:

(1) Contour elements with special geometries (cylindrical, flat and discoid sector);

(2) Analysis of soil-pipeline interaction with the accurate representation of the laminated pipe surface. 


\section{Static Analysis of Buried Pipes Using Coupling between Layerwise Finite Element and Boundary Element Method}

\section{Acknowledgments}

The authors are very grateful to CAPES for the financial support for this research.

\section{References}

[1] Biot, M. A. 1937. "Bending of an Infinite Beam on an Elastic Foundation.” J. App. Mech. Trans. ASME 59: A1-7.

[2] Hetenyi, M. 1946. Beams on Elastic Foundation. Ann Arbor: University of Michigan Press.

[3] Burns, J. Q., and Richard, R. M. 1964. "Attenuation of Stresses for Buried Cylinders." Presented at the Symposium on Soil-Structure Interaction, Engineering Research Laboratory, University of Arizona, Tucson, AZ.

[4] Hoeg, K. 1968. "Stresses against Underground Structural Cylinders." Journal of the Soil Mechanics and Foundations Division 94 (4): 833-58.

[5] Dhar, A. S., Moore, I. D., and McGrath, T. J. 2004. "Two-Dimensional Analyses of Thermoplastic Culvert Deformations and Strains.” Journal of Geotechnical and Geoenvironmental Engineering 130 (2): 199-208.

[6] Spangler, M. G. 1941. "The Structural Design of Flexible Pipe Culverts.” Presented at the Seventeenth Annual Meeting of the Highway Research Board, Washington, USA.

[7] Watkins, R. K., and Spangler, M. G. 1958. "Some Characteristics of the Modulus at Passive Resistance of Soil: A Study of Similitude.” Highway Research Board 3 (7): 576-83.

[8] Greenwood, M. E., and Lang, D. C. 1990. Vertical Deflection of Buried Flexible Pipes, Buried Plastic Pipe Technology. Philadelphia: ASTM STP 1093.

[9] Freitas, J. C. A. 2008. "Boundary Element Method Applied to the Analysis of excavations in Tunnels Using Approximate Dimensional Models.” Master dissertation, Universidade de São Paulo.

[10] Vieira, C. S.2009. "Application of Coupling between the Boundary Element Method and the Finite Element Method for Two-Dimensional Analysis of Soil-Structure Interaction." Ph.D. thesis, Universidade Federal de Alagoas.

[11] Sydenstricker, R. M., and Landau, L. 2000. “A Study of Some Triangular Discrete Reisner-Mindlin Plate and Shell Elements.” Computers and Structures 78: 21-33.

[12] Chen, K. K. 1979. “A Triangular Plate Finite Element for
Large Displacement Elastic-Plastic Analysis of Automobile Structural Components." Computers and Structures 10: 203-15.

[13] Bathe, K. J., and Ho, L. W. 1981. "A Simple and Effective Element for Analysis of General Shell Structures.” Computers and Structures 13: 673-81.

[14] Fafart, M., Dhatt, G., Batoz, J. L. 1989. "A New Descrit Kirchhoff Plate/Shell Element with Procedure.” Computers and Structures 31: 591-606.

[15] Batoz, J. L., Bathe, K. J., and Ho, L. W. 1980. “A Study of Three-Node Triangular Plate Bending Elements.” Int. J. Num. Eng. 15: 1771-812.

[16] Batoz, J. L., and Lardeur, P. 1989. “A Discrete Shear Triangular Nine d.o.f. Element for the Analysis of Thick to Very Thin Plates." International Journal for Numerical Methods in Engineering 28: 533-60.

[17] Sabir, A. B., Sfendji, A., and Hughes, T. G. 1994. "Strain-Based Finite Element for the Natural Frequencies of Cylindrical Shells.” Journal of Thin-Walled Structures 18: 67-82.

[18] Djoudi, M. S., and Bahai, H. 2002. "Strain Based Finite Element for the Vibration of Cylindrical Panels with Openings.” Thin Wall. Struct. 42: 575-88.

[19] Djoudi, M. S., and Bahai, H. 2004. “A Cylindrical Strain-Based Shell Element for Vibration Analysis of Shell Structures.” Fin. Elem. Anal. Desig. 40: 1947-61.

[20] Donnell, L. H. 1938. "A Discussion of Thin Shell Theory." In Proceedings of the Fifth International Congress of Applied Mechanics, 66-70.

[21] Reddy, J. N. 1984. "A Simple High-Order Theory for Laminated Composite Plates.” Journal of Applied Mechanics 51 (4): 745-52.

[22] Barbero, E. J., Reddy, J. N., and Teply, J. L. 1990. "General Two-Dimensional Theory of Laminated Cylindrical Shells.” AIAA Journal 28 (3): 544-53.

[23] Kassegne, S. K. 1992. Layerwise Theory for Discretely Stiffened Laminated Cylindrical Shells. Virginia: Virginia Polytechnic Institute and State University.

[24] Flugge, W. 1973. Stresses in Shells. 2nd ed. Germany: Springer-Verlag.

[25] Menezes R. J. 2008. “On Static and Dynamic Analysis of Problems of Plates and Shells: User-Friendly Environments with Finite Element Method." Graduate Program in Mechanical Engineering, UFPB.

[26] Moser, A. P., and Folkman, S. 2008. Buried Pipe Design. 3rd ed. NY: Mcgraw-Hill. 